\title{
APLICAÇÃO DE MODELOS DE PREVISÃO DE DEMANDA EM UMA FÁBRICA DE EMBALAGENS PLÁSTICAS
}

Eduardo Santos de OLIVEIRA ${ }^{1}$

José Diamantino de Almeida DOURADO²

José André Villas Boas MELLO ${ }^{3}$

\begin{abstract}
${ }^{1}$ Graduação em Engenharia de Produção pelo Centro Federal de Educação Tecnológica Celso Suckow da Fonseca.
${ }^{2}$ Graduação em Engenharia Elétrica/Sistemas de Computação pela Pontifícia Universidade Católica do Rio de Janeiro. Mestrado em Engenharia de Produção pela Pontifícia Universidade Católica do Rio de Janeiro. Doutorado em Análise de Bacias e Faixas Móveis pela Universidade do Estado do Rio de Janeiro. Professor do Centro Federal de Educação Tecnológica Celso Suckow da Fonseca.

${ }^{3}$ Graduação em Ciências Econômicas pela Universidade Federal Rural do Rio de Janeiro. Mestrado em Engenharia de Produção pela Universidade Federal do Rio de Janeiro. Doutorado em Engenharia de Transportes. Professor do Centro Federal de Educação Tecnológica Celso Suckow da Fonseca.
\end{abstract}

\section{Recebido em: 23/10/2016 - Aprovado em: 15/10/2017 - Disponibilizado em: 30/12/2017}

\section{RESUMO:}

Este trabalho tem o objetivo de selecionar, aplicar e avaliar um modelo de previsão que seja o mais adequado às características da demanda do produto investigado e que forneça informações de maior qualidade aos setores produtivos, especialmente ao setor de Planejamento e Controle da Produção (PCP). Como método de pesquisa se optou pelo estudo de caso realizado numa fábrica de embalagens plásticas, buscou-se selecionar um modelo de previsão que fornecesse informações mais precisas ao PCP. Foram escolhidos alguns modelos utilizados atualmente pelas organizações, e os resultados de cada modelo foram confrontados com a demanda real. Identificou o que ofereceu o menor erro ou desvio. Neste estudo de caso, o modelo de Box-Jenkispara a previsão que se baseia no ajuste de modelos de tentativas denominado ARIMA, apresentou resultados com menores taxas de erros, principalmente por ser adequado para as características, tendencias e sazonalidades de demanda do produto principal da empresa.

Palavras-chave:Previsão de demanda, ARIMA, indústria de embalagens plásticas.PCP. Quality.

\begin{abstract}
:
This work aims to select, implementand valuate a forecasting model that is best suited the studied product demand characteristics and to provide higher quality information to the productive sectors, especially to the Planning and Production Control sector (PCP). As a research method, was chosen for the case study conducted in a plastic packaging factory, a prediction model to provide more accurate information to the PCP. We selected some models currently used by the organizations, and the results of each model were confronted with the real demand. Identified what offered theminor error ordeviation. In this case study, the Box-Jenkins model for prediction that is based on the adjustment of tentative models called ARIMA, presented results with lower error rates, mainly because it is adequate for the characteristics, tendencies and seasonality of demand of the main product of the company.
\end{abstract}

Keywords: demandforecasting.ARIMA.Plastic packaging industry. PCP. Quality.

\section{Introdução}

A competição entre as empresas atualmente aumenta a importância a ser dada ao gerenciamento e tratamento das informações referentes ao mercado, concorrentes, economia do país e mundial, investimentos e princiapalmentea demanda por bens e mercadorias.Hastenteufel e Larentis (2015) citam que o mercado contribui para a geração de riqueza da 
empresa e não somente o produto, tornam-se importantes asações de avaliação e gerenciamento para que seja possível adotar novas estratégias na administração dos negócios.

Normalmente as empresas focam somente no tratamento das informações intraempresa, ou seja, informações referentes aos seus processos produtivos, gerenciais ou administrativos como taxas de produtividade, disponibilidade de mão de obra, giro do estoque, demonstrativos contábeis, taxas de juros. No entanto um conhecimento sólido do mercado ao qual a empresa pertence ou dele sofre interferências é, sem dúvidas, o melhor caminho para obter bons resultados, além da permanência no mercado e manutenção de sua competitividade.

Para a realização de uma previsão de demanda existem atualmente diversas ferramentas de auxílio com recursos computacionais, tais como as planilhas eletrônicas e softwares especializados. Tubino (2007) aponta que a previsão de demanda é a base para o planejamento estratégico da produção, vendas e finanças de qualquer empresa. Partindo deste ponto podem-se desenvolver os planos de capacidade, de fluxo de caixa, de vendas, de produção e estoques, de mão-de-obra, e de compras.

É de extrema importância também que as informações obtidas e tratadas na previsão de demanda estejam de acordo com o que a empresa pretende oferecer ao mercado. As atividades de gerenciamento da demanda devem combinar com a estratégia da firma, com as capacidades de produção e com as necessidades do cliente (VOLLMANN et al 2006). A previsão de demanda assume papel central no processo de planejamento da empresa de manufatura. Com base nesta informação, são tomadas as principais decisões financeiras, comerciais e operacionais (LUSTOSA et al, 2008).

Prever a demanda de forma acurada é parte fundamental do processo de Gestão de Demanda das empresas. Como em muitos casos não é possível que a produção seja sob encomenda, há a necessidade de estimar a quantidade de produtos a serem vendidos e utilizar esta previsão (Senna et al., 2015).Pensando na questão da previsão, Moreira et al. (2014) citam que o planejamento e controle da produção é de grande importância para as empresas, pois os planos orientarão a produção e o controle permitirá que os mesmos sejam implementados de forma eficiente.

Este trabalho tem o objetivo de selecionar, aplicar e avaliar um modelo de previsão de demanda que seja o mais adequado às características da demanda do produto escolhido e que forneça informações de maior qualidade aos setores produtivos, especialmente ao setor de Planejamento e Controle da Produção.

$\mathrm{Na}$ indústria, e em seus distintos subsetores, a pesquisa desenvolvida em 
parceria com as universidades pode promover redução de custos e aperfeiçoamento das cadeias produtivas (Felin Nunes et al., 2015).Ao dar importância ao processo de previsão, utilizando-se de dados históricos, pretende-se compreender um gap de pesquisa aplicada que tem sido foco de estudos empresariais que é o comportamento futuro que as vendas possuem, para que o erro entre o valor previsto e o real sejam o menor possível.

\section{ReferencialTeórico}

\subsection{Previsão de demanda}

Para Petrônio e Laugeni(2005) a previsão de demanda é importante para utilizar as máquinas de maneira adequada, para realizar a reposição dos materiais no momento e quantidade certa, e para que todas as demais atividades necessárias no processo industrial sejam adequadamente programadas.

Entende-se por demanda a disposição dos clientes ao consumo de bens e serviços ofertados por uma organização. Essa demanda é influenciada por uma série de fatores que se estendem desde as condições macroeconômicas até questões operacionais, como a disponibilidade do produto e preço no ponto-de-venda (LUSTOSA et al., 2008).

O processo de previsão de demanda pode ser classificado em quantitativa ou qualitativa, dependendo do tipo de modelo ou processo utilizado. Enquanto a primeira baseia-se em modelos numéricos, modelos matemáticos e estatísticos, a última utiliza da experiência, opiniões e conhecimento dos tomadores de decisões com relação ao mercado.

Os modelos qualitativos de previsão, também chamados de intuitivos ou subjetivos, dependem da experiência acumulada pelos especialistas. As técnicas qualitativas privilegiam principalmente dados subjetivos(LEMOS, 2006).Os métodos qualitativos apresentam um maior grau de subjetividade e, por isso, parecem menos adequados que os qualitativos. No entanto, quando não há disponibilidade de dados, tornam-se a única referência (LUSTOSA et al. 2008).

Ainda segundo Lustosa et al. (2008), alguns métodos ou modelos qualitativos são:

- Pesquisa de mercado: Busca-se avaliar a demanda potencial de um produto ou serviços diretamente com os consumidores finais, através de diferentes métodos de pesquisa;

- Simulação de cenários: Busca-se construir a partir da opinião de especialistas, diferentes cenários futuros e, para cada um deles, estimar o comportamento das vendas;

- Método Delphi: A opinião de especialistas pode ser usada na previsão e estimativa quando não se há possibilidade de serem obtidas através de outro método. É um processo rápido e barato, porém não 
permite utilizar o potencial da análise estatística dos dados, não sendo possível controlar os erros nem os vieses.

Para Correa et al. (2008) os modelos quantitativos baseiam-se em métodos estatísticos, com diferentes características e níveis de complexidade. Os principais modelos estatísticos de previsão são: Projeção de séries temporais e correlação e regressão.Nos modelos de séries temporais, considera-se que a variável demanda é função apenas da variável tempo. Por outro lado os modelos de correlação e regressão, a variável demanda pode estar relacionada com outras variáveis independentes.

\subsection{Método das médias móveis}

Considera a média aritmética de " $n$ " períodos anteriores, buscando, dessa forma, suavizar os resultados da previsão. Á medida que um novo valor é incorporado à série, o valor mais antigo é descartado (LUSTOSA et al 2008). Matematicamente é determinado pela equação 1:

$F_{t}(t+k)=\left(D_{t}+D_{t-1}+\cdots+D_{t-n+1}\right) /$

$n k=1,2, \ldots$

Onde:

$\mathrm{F}_{\mathrm{t}}$ - previsão de demanda no instante $\mathrm{t}$;

$D_{t}$ - demanda no instante $t$;

n - quantidade de períodos considerada

Os métodos de médias móveis são adequados para as séries históricas de vendas que não apresentam tendência ou sazonalidade. "Recomenda-se o uso da média móvel em situações nas quais a demanda apresenta comportamento estável e o produto não é muito relevante (TUBINO, 2007).”

Segundo Morettin e Toloi (2006) as principais vantagens deste método são:

1. Simples aplicação;

2. É aplicável quando se tem um número pequeno de observações.

Como desvantagem do método de média móvel tem-se:

1. Deve ser utilizado para prever somente séries estacionárias;

2. Dificuldade em determinar a quantidade de meses utilizados para a média.

\subsection{Método de Box Jenkins(ARIMA)}

ARIMA é o nome dado ao modelo criado em 1976 pelos estatísticos George Box e GwilymJenkins, também conhecido como Modelo de Box-Jenkins. O nome ARIMA deriva do inglês autoregressiveintegratedmovingaverage, ou seja, modelo autoregressivo integrado de médias móveis. È uma generalização do modelo ARMA, modelo autoregressivo de média móvel. Representa-se na forma $\operatorname{ARIMA}(\mathrm{p}, \mathrm{d}, \mathrm{q})$, onde:

- $\mathrm{p}$ é o número de termos autoregressivos;

- d é o número de diferenças e;

- q é o número de termos da média móvel. 
O modelo autoregressivo de média móvel, ARIMA (p,0,q), é um modelo que utiliza a combinação dos modelos autoregressivos, ou $\mathrm{AR}(\mathrm{p})$, e modelos de médias móveis, ou MA(q) (FAVA, 2000). No modelo $\mathrm{AR}(\mathrm{p})$ o valor do processo é expresso como um agregado linear dos valores anteriores e a adição dos distúrbios $\varepsilon_{\mathrm{t}}(\mathrm{GALVÍNCIO}$ et al, 2002).No caso de combinação dos modelos, o valor de y em determinado período de tempo $\mathrm{t}$ é descrito pelos seus valores passados e pelos choques aleatórios constantes e passados, que são os pressupostos dos modelos $\mathrm{AR}(\mathrm{p})$ e $\mathrm{MA}(\mathrm{q})$ respectivamente (FAVA, 2000).

A equação genérica de um modelo $\operatorname{ARIMA}(\mathrm{p}, \mathrm{d}, \mathrm{q})$ utiliza-se de um filtro $\operatorname{AR}(\mathrm{p})$, representado pelo segundo termo na equação 3.

$$
\begin{gathered}
Y_{t}=\emptyset Y_{t-1}+\cdots+\emptyset_{p} Y_{t-p}+\varepsilon_{t}-\theta_{1} \varepsilon_{t-1}- \\
\cdots-\theta_{q} \varepsilon_{t-q}
\end{gathered}
$$

Onde:

$\mathrm{Yt}$ - valor previsto no instante $\mathrm{t}$;

Ǿ - operador auto-regressivo de ordem p;

$\theta$ - operador de médias-móveis de ordem q;

$\varepsilon$ - erro ou ruído branco.

A primeira exigência para se utilizar o modelo ARIMA é que a série seja estacionária ou possa ser transformada. Uma série estacionária é aquela que possui média constante e não possui tendência no decorrer do tempo. A análise gráfica da série normalmente já possibilita a identificação da estacionariedade da série.
Como dito acima, o modelo ARIMA é formado pela integração entre os modelos $\operatorname{AR}(p)$ e $M A(q)$. Os modelosAR(1), $\operatorname{AR}(2)$ e AR(3) podem ser escritos da seguinte forma:

$\operatorname{AR}(1): Y_{t}=d+\varnothing(1) * Y_{t-1}+\varepsilon_{t}(4)$

$\operatorname{AR}(2): Y_{t}=d+\emptyset(1) * Y_{t-1}+\emptyset(2) *$

$Y_{t-2}+\varepsilon_{t}$

AR (3): $Y_{t}=d+\emptyset(1) * Y_{t-1}+\emptyset(2) *$

$Y_{t-2}+\emptyset(3) * Y_{t-3}+\varepsilon_{t}$

Onde:

d - Constante ou termo de tendência;

Um modelo MA(q) é uma média móvel ponderada, de número fixo, de erros de previsões, produzidos no passado, por isso é chamado média móvel. Diferentemente da média móvel tradicional, os pesos numa MA(q) não são iguais e não somam 1 (BERTOLO, 2010). Os modelos MA(1), MA(2) e MA(3) podem ser escritos da seguinte forma:

$\operatorname{MA~(1):~} Y_{t=} e_{t}+\theta(1) * e_{(t-1)}(7)$

$\operatorname{MA~(2):~} Y_{t}=e_{t}+\theta(1) * e_{(t-1)}+\theta(2) *$

$e_{(t-2)}$

MA (3): $Y_{t}=e_{t}+\theta(1) * e_{(t-1)}+\theta(2) *$

$e_{(t-2)}+\theta(3) * e_{(t-3)}(9)$

Unindo os modelos $\mathrm{AR}(\mathrm{p})$ e $\mathrm{MA}(\mathrm{q})$, obtém-se a equação (3), ou seja, o modelo ARIMA (p,d,q). Caso a série não seja estacionária, deve-se diferenciá-la tantas vezes forem até obter uma série estacionária, representados pelo parâmetro d. Na maioria dos casos usuais, $d=1$ ou $d=2$, que correspondem a dois casos comuns não- 
estacionariedade homogênea (MORETTIN e TOLLOI, 2006).

\subsection{Erros das previsões}

O indicador básico de erro de previsão para o período $t\left(E_{t}\right)$ é a diferença(ou desvio) entre o valor real $\left(D_{t}\right)$ e o valor previsto da demanda $\left(\mathrm{F}_{\mathrm{t}}\right.$, forecast $)$ no período correspondente. Desvios positivos significam que a demanda superou a previsão, e negativos, o contrário (LUSTOSA et al., 2008). O erro é dado por:

$E_{t}=D_{t}-F_{t}$

O erro médio é dado por:

$E M=\sum_{t=1}^{n}\left(D_{t}-F_{t}\right) / n$

Para a análise e conclusões obtidas com este trabalho será utilizado como principal tipo de erro o MAPE, erro percentual absoluto médio.

$M A P E=\sum_{t=1}^{n}|D t-F t| /|D t| / n$

\section{Materiais e Métodos}

A metodologia adotada é a baseada em pesquisa bibliográfica, científica, referente ao assunto, e estudo de caso, onde buscará através das ferramentas e modelos levantados com a pesquisa, propor a adoção de um modelo a fim de solucionar a dificuldade da empresa na previsão de demanda.

Esta pesquisa está classificada, conforme Silva e Menezes (2005) em aplicada e quantitativa. A pesquisa é aplicada quando gera conhecimento para aplicação prática a solução de problemas específicos; É quantitativa quando pode ser traduzida em números, com a utilização de técnicas estatísticas e modelagem.

Também classificamos esta pesquisa em exploratória e estudo de caso. Segundo Gil (1999) a pesquisa é exploratória quando visa proporcionar maior familiaridade com o problema com vistas a torná-lo explícito ou a construir hipóteses; É um estudo de caso quando envolve o estudo profundo e exaustivo de um ou poucos objetos de maneira que se permita o seu amplo e detalhado conhecimento.

A definição do ambiente de pesquisa e os indivíduos considerados é fator fundamental para o levantamento das informações do problema em estudo.Para Gil (1999) universo é o conjunto definido de elementos que possuem determinadas características, sendo a amostra um subconjunto do universo ou população, em que se estabelecem as características deste universo ou população.

Desta forma o universo ou população da pesquisa são os dados históricos de vendas, delimitados pelo horizonte de tempo a ser determinado, este determinado em função do modelo de previsão escolhido. As análises realizadas neste trabalho, bem como os gráficos, foram feitas através do software Minitab®, desenvolvido pela MinitabInc., versão gratuita por 30 dias. Esta versão esta 
disponível para download em

www.minitab.com.br. Para a montagem das planilhas foi utilizado o software Open Office $^{\mathrm{TM}}$, desenvolvido pela The Apache Software Foundation. Para este software não é necessário licença, e seu download pode ser realizado em www.openoffice.com.br.

O modelo que a empresa utilizafoi posto como referência, ou seja, o modelo de médias móveis com horizonte de 12 meses. A previsão gerada por este modelo foi utilizada como base para a seleção do modelo ARIMA que mais se adequará as características da demanda desta empresa. Para efeitos de comparação serão aplicados 4 variantes do modelo aos dados históricos, ou seja, será utilizado o modelo ARIMA(p,d,q) em que os parametros $\mathrm{p}, \mathrm{d}$ e q terão valores diferentes.

Os dados serão obtidos basicamente por consulta ao módulo de faturamento no sistema ERP da empresa, para o período referente a janeiro de 2007 a dezembro de 2012, informações obtidas pelos gestores das áreas envolvidas na tomada de decisão do processo de previsão além de outros eventos que podem influenciar a demanda. Sendo assim o processo de obtenção de dados se dará através da seguinte estrutura, proposta por Lemos (2005):

(i) Discriminação dos níveis industriais de previsão;

(ii) Definição dos fatores temporais;

(iii) Priorização dos itens a serem previstos; (iv) Análise do ciclo de vida dos itens a serem previstos;

(v) Definição dos dados de entrada e saída;

(vi) Coleta e preparação dos dados.

Os dados históricos de vendas referem-se ao produto A, o principal da empresa, e foram coletados com base na curva $\mathrm{ABC}$, correspondente ao período de janeiro de 2007 a dezembro de 2012. Os modelos serão aplicados para realizar comparação entre as previsões referentes ao ano de 2012 e a demanda real, e assim ser feita a seleção do modelo mais apropriado. Para a aplicação e comparação dos métodos foi escolhido somente este produto, de uma determinada família e grupo, mas que representa perfeitamente o comportamento da demanda global. O nome deste produto e sua aplicação não serão mencionados a pedido da empresa.

\section{Resultados}

O estudo de caso foi realizado em uma fábrica de embalagens plásticas no Estado do Rio de Janeiro, baixada fluminense. Esta fábrica está em atividade há aproximadamente 40 anos e seus produtos são tradicionais no mercado. O elemento diferencial de seus produtos com relação aos concorrentes é o alto nível de qualidade. Além de atender ao mercado interno, alguns de seus produtos também são exportados, com um número de vendas crescente a cada mês.A demanda de seus produtos, normalmente não sofre com a 
sazonalidade de outros setores da economia, pois são produtos de necessidades básicas, utilizados na coleta de lixo residencial e industrial, porém suas características dificultam a previsão de demanda obtida com modelos gerais, como as médias móveis.

A empresa utiliza este modelo para realizar suas previsões, porém percebeu-se através do setor de PCP que a diferença entre a demanda prevista e a realizada no produto $\mathrm{A}$ estava com margem de erro muito grande, denotando um risco à produção e ao cumprimento do prazo de entrega dos pedidos, além dos custos extras de produção por falta de material, horas extras, frete e etc.

Com base na dificuldade relatada, serão estudados alguns modelos de previsão de demanda, com a aplicação deles no cenário encontrado pela empresa, e após compararemos entre si seus erros de previsão, para encontrarmos o modelo que fornece melhores resultados. Entende-se como melhores resultados o modelo que se aproxima mais da demanda do produto A, e não necessariamente um modelo que apresente menor erro com relação a demanda efetiva.
O produto escolhido, para a seleção do modelo de previsão mais adequado, encontrase atualmente na fase de maturação, ou estabilidade, e não há indícios de que esteja entrando na fase de declínio. Apesar de o produto estar no mercado a mais de 20 anos, percebe-se pelas suas características de uso doméstico e industrial, que continuará por muito tempo na fase de estabilidade. Desta forma, o modelo de previsão selecionado dificilmente sofrerá influência de uma mudança de fase do produto.

\subsection{Aplicação dos modelos}

Inicialmente se aplicou o modelo de médias móveis de 12 meses, utilizado pelaempresa utiliza. A previsão gerada por este modelo foi utilizada como base para a seleção do modelo que mais se adequará as características da demanda desta empresa.

Na figura 1, se apresenta o gráfico da curva de demanda do produto A para o estudo e na tabela 5 os valores referentes às 60 (Sessenta) observações de vendas, do período de janeiro de 2007 a dezembro de 2011. Pretende-se gerar a previsão da demandautilizando este período com os modelos e comparar seus erros. 


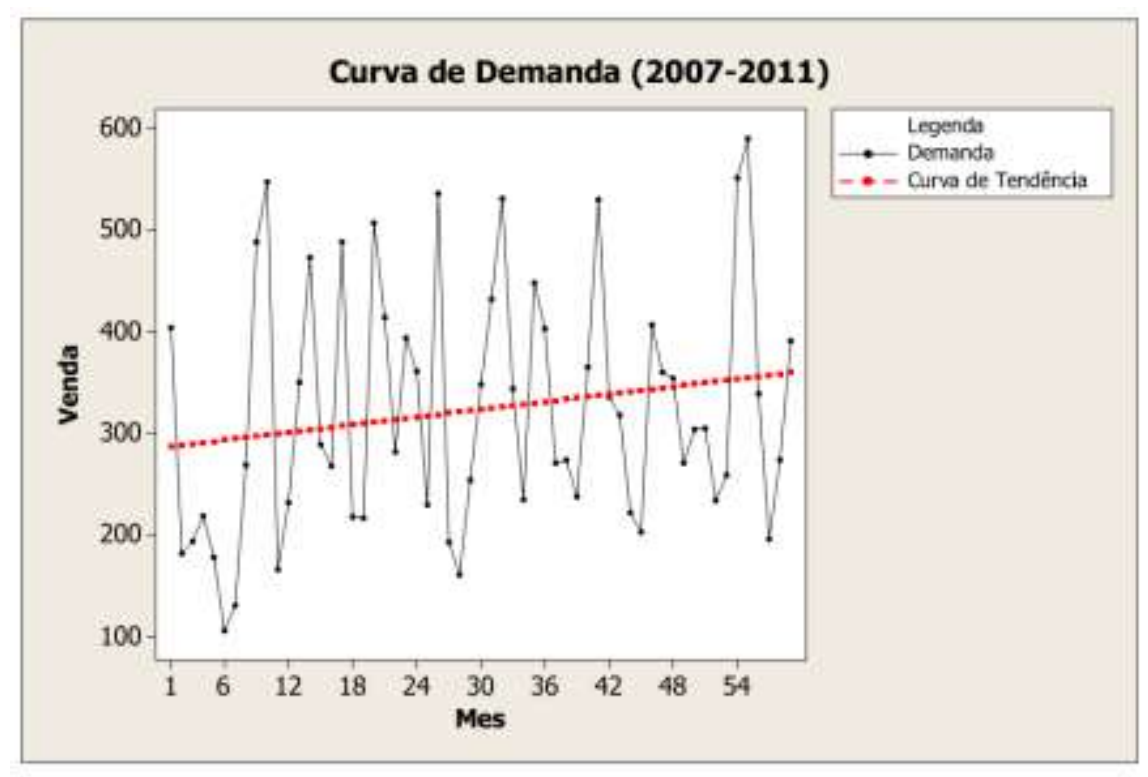

Figura 1 - Curva de demanda janeiro de 2007 a dezembro de 2011.

Observa-se na figura 1 que a demanda não apresenta estacionariedade, possui tendência e variabilidade acentuada, ou seja, vários períodos de sazonalidade, vide tabela 1 ,fatores determinantes para a escolha do modelo de previsão de demanda apropriado.

Tabela 1: Observações mensais de vendas 2007 a 2011.

\begin{tabular}{|c|c|c|c|c|c|c|c|c|c|}
\hline Mês & Vendas & Mês & Vendas & Mês & Vendas & Mês & Vendas & Mês & Vendas \\
\hline 1 & 350 & 13 & 230 & 25 & 271 & 37 & 271 & 49 & 513 \\
\hline 2 & 473 & 14 & 536 & 26 & 274 & 38 & 173 & 50 & 275 \\
\hline 3 & 289 & 15 & 193 & 27 & 238 & 39 & 131 & 51 & 360 \\
\hline 4 & 268 & 16 & 161 & 28 & 365 & 40 & 305 & 52 & 201 \\
\hline 5 & 488 & 17 & 254 & 29 & 530 & 41 & 234 & 53 & 236 \\
\hline 6 & 218 & 18 & 348 & 30 & 336 & 42 & 259 & 54 & 127 \\
\hline 7 & 217 & 19 & 432 & 31 & 318 & 43 & 551 & 55 & 117 \\
\hline 8 & 507 & 20 & 531 & 32 & 222 & 44 & 590 & 56 & 158 \\
\hline 9 & 414 & 21 & 344 & 33 & 203 & 45 & 339 & 57 & 278 \\
\hline 10 & 282 & 22 & 235 & 34 & 407 & 46 & 196 & 58 & 98 \\
\hline 11 & 394 & 23 & 448 & 35 & 360 & 47 & 274 & 59 & 202 \\
\hline 12 & 361 & 24 & 403 & 36 & 354 & 48 & 391 & 60 & 284 \\
\hline
\end{tabular}


Para o método de médias móveis, utilizamos uma série histórica de 12 meses, de janeiro a dezembro de 2011, para projetar 12 meses seguintes, representados na figura 2 . $\mathrm{Na}$ tabela, gerada pelo software Minitab ${ }^{\circledR}$, podemos observar a demanda, representada pela linha em cor preta e os valores previstos, na cor vermelha. Também obtemos os erros (MAPE, MAD e MSD), além do tamanho da média móvel.

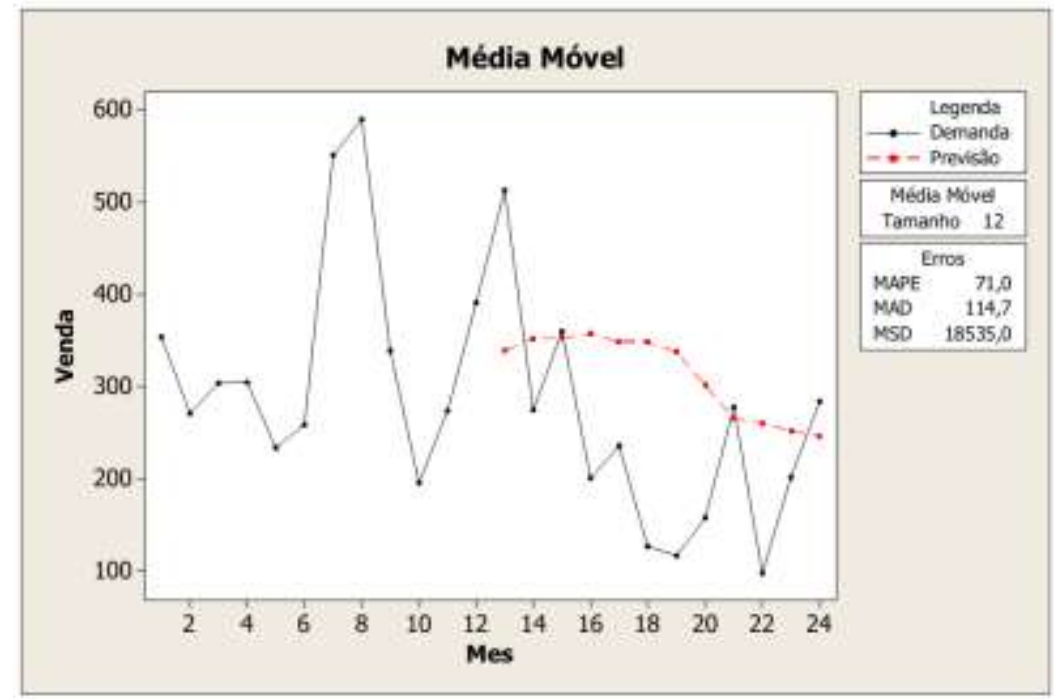

Figura 2 - Média móvel 12 meses

Na tabela 2 temos a memória de cálculo do modelo de médias-móveis com 12 meses. Conforme funcionamento do modelo, os valores de previsão só existem a partir do $12^{\circ}$ mês, já que o modelo necessita destes valores para projetar o primeiro mês. Na tabela 3 temos os erros gerados pelo Minitab $^{\circledR}$, onde vemos o erro percentual absoluto em $71 \%$, ou seja, somente $29 \%$ de chance de a previsão obtida com este modelo atender a demanda.

Tabela 2: Demanda prevista com média móvel 12 meses.

\begin{tabular}{|c|c|c|c|c|c|c|c|}
\hline Mês & Demanda & Previsão & Erro & Mês & Demanda & Previsão & Erro \\
\hline jan/11 & 354 & & & jan/12 & 513 & 352 & 161 \\
\hline fev/11 & 271 & & & fev/12 & 275 & 353 & -78 \\
\hline mar/11 & 304 & & & mar/12 & 360 & 357 & 3 \\
\hline abr/11 & 305 & & & abr/12 & 201 & 349 & -148 \\
\hline mai/11 & 234 & & & mai/12 & 236 & 349 & -113 \\
\hline jun/11 & 259 & & & jun/12 & 127 & 338 & -211 \\
\hline
\end{tabular}




\begin{tabular}{|c|c|c|c|c|c|c|c|} 
jul/11 & 551 & & & jul/12 & 117 & 302 & -185 \\
\hline ago/11 & 590 & & & ago/12 & 158 & 266 & -108 \\
\hline set/11 & 339 & & & set/12 & 278 & 261 & 18 \\
\hline out/11 & 196 & & & out/12 & 98 & 252 & -154 \\
\hline nov/11 & 274 & & & nov/12 & 202 & 246 & -44 \\
\hline dez/11 & 391 & 339 & 52 & dez/12 & 284 & 237 & 47 \\
\hline
\end{tabular}

Tabela 3: Medidas de Acurácia do modelo média móvel com 12 meses.

\begin{tabular}{|l|c|}
\hline \multicolumn{2}{|c|}{ Erros } \\
\hline MAPE & 71 \\
\hline MAD & 114,7 \\
\hline MSD & 18535 \\
\hline
\end{tabular}

Para as projeções com base na simples, duplo e de Winters, conforme figuras suavização exponencial, utilizamos os 3, 8,9 e 10. métodos de suavizamento exponencial

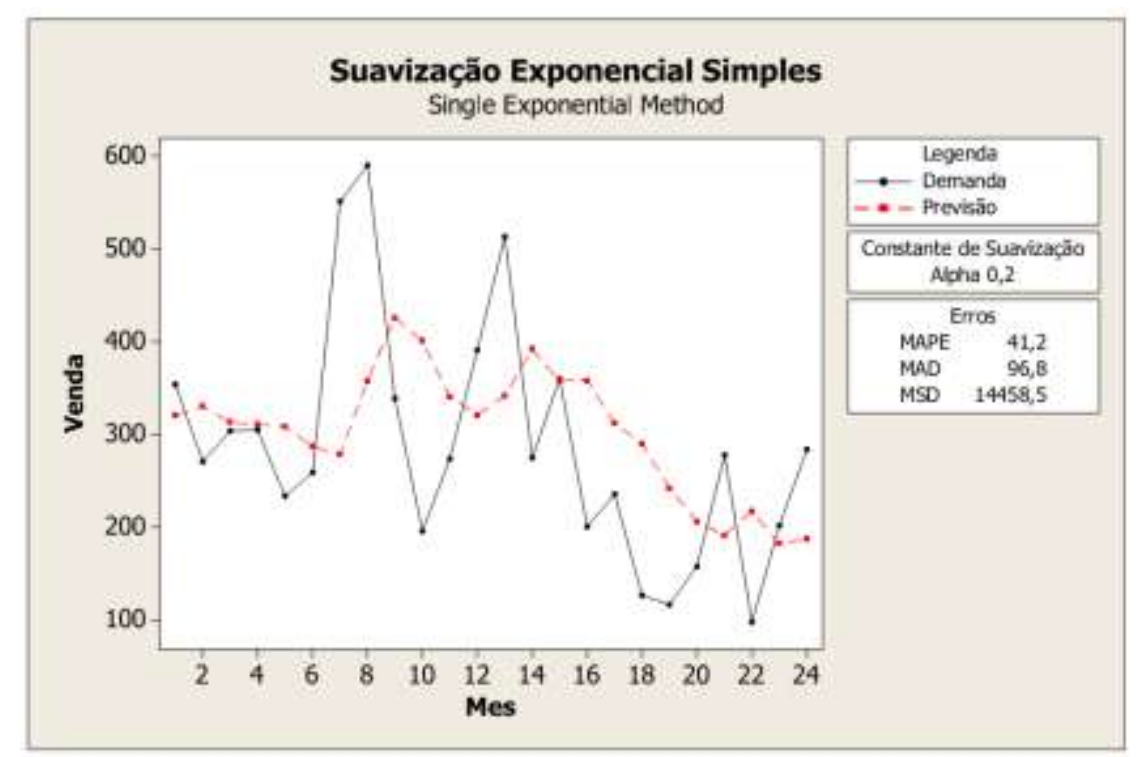

Figura 3 - Suavização exponencial simples

$\mathrm{Na}$ tabela 4 vemos o resultado da aplicação do método de suavização exponencial simples, com constante de suavização igual a 0,2 , que atribui pesos menores aos valores mais antigos da série histórica. 
Tabela 4: Demanda prevista com método de suavização exponencial simples.

\begin{tabular}{|c|c|c|c|c|c|c|c|}
\hline Mês & Demanda & Previsão & Erro & Mês & Demanda & Previsão & Erro \\
\hline jan/11 & 354 & 301 & 53 & jan/12 & 513 & 374 & 139 \\
\hline fev/11 & 271 & 295 & -24 & fev/12 & 275 & 355 & -80 \\
\hline mar/11 & 304 & 297 & 7 & mar/12 & 360 & 356 & 4 \\
\hline abr/11 & 305 & 298 & 7 & abr/12 & 201 & 325 & -124 \\
\hline mai/11 & 234 & 286 & -52 & mai/12 & 236 & 307 & -71 \\
\hline jun/11 & 259 & 280 & -21 & jun/12 & 127 & 271 & -144 \\
\hline jul/11 & 551 & 334 & 217 & jul/12 & 117 & 240 & -123 \\
\hline ago/11 & 590 & 386 & 204 & ago/12 & 158 & 224 & -66 \\
\hline set/11 & 339 & 376 & -37 & set/12 & 278 & 235 & 43 \\
\hline out/11 & 196 & 340 & -144 & out/12 & 98 & 207 & -109 \\
\hline nov/11 & 274 & 327 & -53 & nov/12 & 202 & 206 & -4 \\
\hline dez/11 & 391 & 340 & 51 & dez/12 & 284 & 222 & 62 \\
\hline
\end{tabular}

Conforme erros, calculados pelo Minitab $^{\circledR}$, tabela 5, se vê que o modelo de suavização exponencial já apresenta MAPE muito menor que no modelo de médias móveis. Com a constante de suavização, $\alpha$, igual a 0,2 , temos $20 \%$ do valor real mais recente e $80 \%$ do valor da previsão mais recente. Com base nesta relação, valor real mais recente e previsão mais recente, obtemos o valor de $\alpha$ mais apropriado, porém com $\alpha$ igual a 0,2 já temos uma previsão melhor do que o fornecido pelo modelo anterior.

Tabela 5: Medidas de acurácia do modelo de suavização exponencial simples.

\begin{tabular}{|l|c|}
\hline \multicolumn{2}{|c|}{ Erros } \\
\hline MAPE & 42,2 \\
\hline MAD & 95,8 \\
\hline MSD & 14761 \\
\hline
\end{tabular}

Uma variante dos modelos de suavização, a exponencial dupla, apresentada na figura 4 também fornece bons resultados, próximos aos valores da suavização simples. além de $\beta$ igual a 0,2. Este modelo, ao Neste caso também se utilizou $\alpha$ igual a 0,2 , 


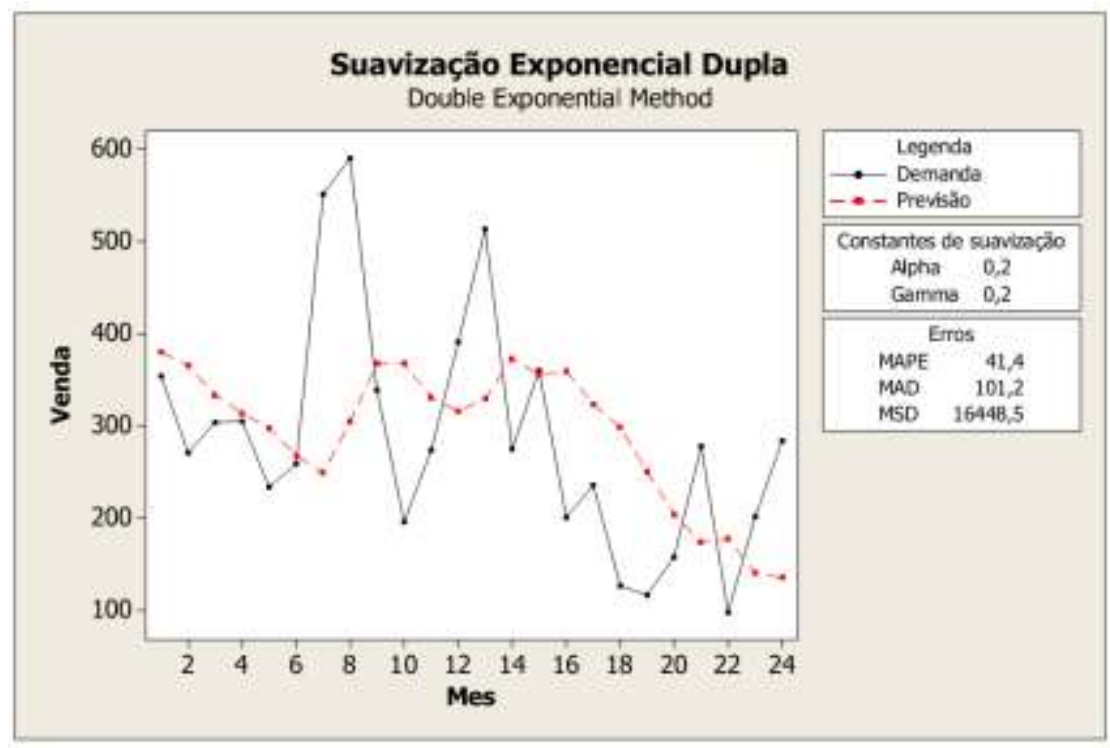

Figura 4 - Suavização exponencial dupla

Na tabela 6 e 7 se tem o resultado da aplicação do método de suavização exponencial dupla, com constante de suavização igual a 0,2 , que atribui pesosmenores aos valores mais antigos da série histórica.

Tabela 6: Demanda prevista com suavização exponencial dupla.

\begin{tabular}{|c|c|c|c|c|c|c|c|}
\hline Mês & Vendas & Previsão & Erro & Mês & Vendas & Previsão & Erro \\
\hline jan/11 & 354 & 380 & -26 & jan/12 & 513 & 330 & 183 \\
\hline fev/11 & 271 & 366 & -95 & fev/12 & 275 & 373 & -98 \\
\hline mar/11 & 304 & 334 & -30 & mar/12 & 360 & 356 & 4 \\
\hline abr/11 & 305 & 314 & -9 & abr/12 & 201 & 359 & -158 \\
\hline mai/11 & 234 & 298 & -64 & mai/12 & 236 & 324 & -88 \\
\hline jun/11 & 259 & 268 & -9 & jun/12 & 127 & 299 & -172 \\
\hline jul/11 & 551 & 249 & 302 & jul/12 & 117 & 250 & -133 \\
\hline ago/11 & 590 & 304 & 286 & ago/12 & 158 & 204 & -46 \\
\hline set/11 & 339 & 368 & -29 & set/12 & 278 & 174 & 104 \\
\hline out/11 & 196 & 367 & -171 & out/12 & 98 & 177 & -79 \\
\hline nov/11 & 274 & 331 & -57 & nov/12 & 202 & 141 & 61 \\
\hline dez/11 & 391 & 316 & 75 & dez/12 & 284 & 136 & 148 \\
\hline
\end{tabular}


Tabela 7: Medidas de acurácia suavização exponencial dupla.

\begin{tabular}{|l|c|}
\hline \multicolumn{2}{|c|}{ Erros } \\
\hline MAPE & 41,4 \\
\hline MAD & 101,2 \\
\hline MSD & 16448,5 \\
\hline
\end{tabular}

$\mathrm{Na}$ figura 5 temos o gráfico da previsão de demanda realizada com o método de suavização de Winters. Este modelo é mais apropriado quando precisamos tratar simultaneamente as componentes da tendência e sazonalidade. Este modelo trabalha na forma multiplicativa ou aditiva.

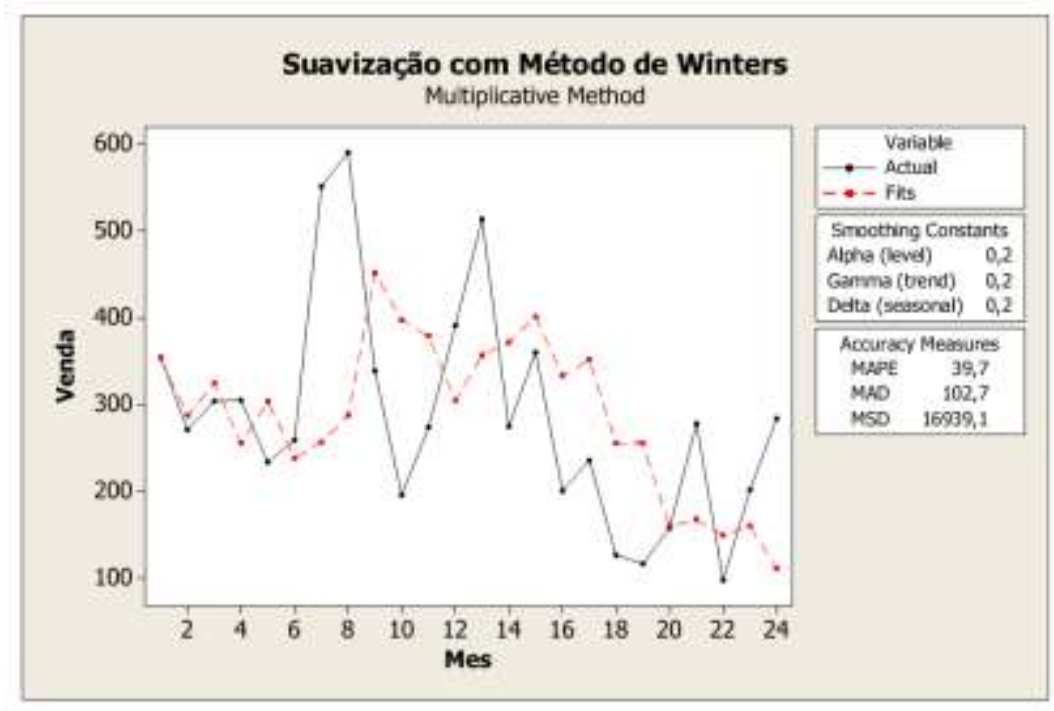

Figura 5 - Suavização exponencial de Winters (Método Multiplicativo)

$\mathrm{Na}$ tabela 8 vemos o resultado da aplicação do método de suavização exponencial de winters, com constante de suavização igual a 0,2, que atribui pesos menores aos valores mais antigos da série histórica.

Tabela 8: Demanda prevista com suavização exponencial de Winters (Método multiplicativo).

\begin{tabular}{|c|c|c|c|c|c|c|c|}
\hline Mês & Venda & Previsão & Erro & Mês & Venda & Previsão & Erro \\
\hline jan/11 & 354 & 354 & 0 & jan/12 & 513 & 357 & 156 \\
\hline fev/11 & 271 & 287 & -16 & fev/12 & 275 & 372 & -97 \\
\hline mar/11 & 304 & 325 & -21 & mar/12 & 360 & 401 & -41 \\
\hline abr/11 & 305 & 255 & 50 & abr/12 & 201 & 333 & -132 \\
\hline
\end{tabular}




\begin{tabular}{|c|c|c|c|c|c|c|c|} 
mai/11 & 234 & 304 & -70 & $\mathrm{mai} / 12$ & 236 & 352 & -116 \\
\hline $\mathrm{jun} / 11$ & 259 & 239 & 20 & $\mathrm{jun} / 12$ & 127 & 255 & -128 \\
\hline $\mathrm{jul} / 11$ & 551 & 257 & 294 & $\mathrm{jul} / 12$ & 117 & 256 & -139 \\
\hline $\mathrm{ago} / 11$ & 590 & 287 & 303 & $\mathrm{ago} / 12$ & 158 & 160 & -2 \\
\hline set/11 & 339 & 452 & -113 & $\mathrm{set} / 12$ & 278 & 168 & 110 \\
\hline out/11 & 196 & 397 & -201 & $\mathrm{out} / 12$ & 98 & 150 & -52 \\
\hline nov/11 & 274 & 378 & -104 & nov/12 & 202 & 160 & 42 \\
\hline dez/11 & 391 & 304 & 87 & $\mathrm{dez} / 12$ & 284 & 112 & 172 \\
\hline
\end{tabular}

No modelo multiplicativo o nível da série é multiplicado por uma componente sazonal, fazendo com que o nível médio aumente com o passar do tempo, devido asazonalidade. No caso do modelo aditivo também há o aumento do nível médio devido a componente de sazonalidade, porém mantém-se constante a amplitude da flutuação em relação ao nível médio.

Tabela 9: Medidas de acurácia suavização exponencial de Winters (Método Multiplicativo).

\begin{tabular}{|l|c|}
\hline \multicolumn{2}{|c|}{ Erros } \\
\hline MAPE & 39,7 \\
\hline MAD & 102,7 \\
\hline MSD & 16939,1 \\
\hline
\end{tabular}

Como se pode observar a suavização exponencial de Winters, também conhecida como Holt-Winters, apresenta um erro ligeiramente menor. Observação feita para o modelo de suavização simples, com relação a obtenção dos valores ótimos das constantes de suavização, também é válida para este modelo. No entanto uma previsão feita com valores de $\alpha=\beta=0,2$ já apresenta resultados muito melhores dos que os fornecidos pela média móvel, modelo utilizado atualmente pela empresa.
Nautilizaçãodos modelos ARIMA $(1,1,1), \quad \operatorname{ARIMA}(2,1,1), \quad \operatorname{ARIMA}(3,2,2) \quad \mathrm{e}$ ARIMA (3,1,3). O primeiro parâmetro, p, refere-se à quantidade de termos autoregressivos utilizados para a previsão. $\mathrm{O}$ segundo parâmetro do modelo, $d$, representa a quantidade de diferenças aplicadas na série original, a fim de se obter uma série estacionária, já que a aplicação destes modelos pressupõe-se que há estacionariedade na série. 
Em um único modelo utilizamos o parâmetro d igual a 2, pois o software informou que o modelo $\operatorname{ARIMA}(3,1,1)$ ajustado gerou erro por não ser nãoestacionário ou não-invertível. Por fim o termo q refere-se à quantidade de termos da média-móvel. Na tabela 10 se apresentam os resultados do ano de 2012 decada modelo ARIMA utilizado no Minitab ${ }^{\circledR}$, bem como se apresentam na tabela 11 os seus erros.

Tabela 10: Previsão com método ARIMA.

\begin{tabular}{|c|c|c|c|c|c|c|c|c|c|}
\hline Mês & Venda & $\begin{array}{c}\text { Previsã } \\
\text { o } \\
\text { ARIM } \\
\text { A } \\
(1,1,1)\end{array}$ & $\begin{array}{c}\text { Erro } \\
\text { ARIMA } \\
(\mathbf{1 , 1 , 1 )}\end{array}$ & $\begin{array}{c}\text { PrevisãoARI } \\
\text { MA }(2,1,1)\end{array}$ & $\begin{array}{c}\text { ErroARIM } \\
\text { A }(2,1,1)\end{array}$ & $\begin{array}{c}\text { PrevisãoARI } \\
\text { MA }(\mathbf{3 , 2 , 2})\end{array}$ & $\begin{array}{c}\text { ErroARIM } \\
\text { A }(\mathbf{3 , 2 , 2 )}\end{array}$ & $\begin{array}{c}\text { Previsã } \\
\text { o } \\
\text { ARIM } \\
\text { A } \\
(3,2,2)\end{array}$ & $\begin{array}{c}\text { Erro } \\
\text { ARIM } \\
\text { A } \\
(3,2,2)\end{array}$ \\
\hline $\mathrm{jan} / 12$ & 513 & 313 & 200 & 338 & 175 & 335 & 178 & 344 & 169 \\
\hline fev/12 & 275 & 343 & -68 & 339 & -64 & 563 & -288 & 333 & -58 \\
\hline $\mathrm{mar} / 12$ & 360 & 290 & 70 & 231 & 129 & 290 & 70 & 215 & 145 \\
\hline abr/12 & 201 & 308 & -107 & 335 & -134 & 483 & -282 & 366 & -165 \\
\hline mai/12 & 236 & 271 & -35 & 257 & -21 & 10 & 226 & 207 & 29 \\
\hline jun/12 & 127 & 276 & -149 & 315 & -188 & 266 & -139 & 317 & -190 \\
\hline jul/12 & 117 & 248 & -131 & 265 & -148 & 35 & 82 & 233 & -116 \\
\hline ago/12 & 158 & 241 & -83 & 289 & -131 & 77 & 81 & 244 & -86 \\
\hline set/12 & 278 & 246 & 32 & 296 & -18 & 170 & 108 & 311 & -33 \\
\hline out/12 & 98 & 271 & -173 & 313 & -215 & 231 & -133 & 251 & -153 \\
\hline nov/12 & 202 & 227 & -25 & 215 & -13 & 117 & 85 & 226 & -24 \\
\hline $\mathrm{dez} / 12$ & 284 & 247 & 37 & 299 & -15 & 278 & 6 & 267 & 17 \\
\hline
\end{tabular}

Tabela 11: Medidas de Acurácia com método ARIMA.

\begin{tabular}{|c|c|c|c|c|}
\hline & $\begin{array}{c}\text { ERROS } \\
\text { ARIMA } \\
(\mathbf{1 , 1 , 1})\end{array}$ & $\begin{array}{c}\text { ERROS } \\
\text { ARIMA } \\
\mathbf{( 2 , 1 , 1 )}\end{array}$ & $\begin{array}{c}\text { ERROSARIMA } \\
\mathbf{( 3 , 2 , 2 )}\end{array}$ & $\mathbf{( 3 , 1 , 3 )}$ \\
\hline MAPE & 32,7 & $\mathbf{3 1 , 1}$ & 49,0 & 29,7 \\
\hline MAD & 98,1 & $\mathbf{8 9 , 1}$ & 142,2 & 87,7 \\
\hline MSD & 16149,7 & $\mathbf{1 4 8 0 6 , 6}$ & 30801,9 & 13448,0 \\
\hline
\end{tabular}

\subsection{Comparação dos resultados}

Todos os resultados de erros dos modelos são unificadasa fim de verificar qual modelo apresentou menores erros, conforme tabela 12.É possível observar que à medida que aplicamos modelos com complexidade 
crescente, ou seja, do modelo de médiasapresentou queda significativa em geral. móveis até os modelos ARIMA, o erro MAPE

Tabela 12: Resultado geral dos modelos de previsão de demanda.

\begin{tabular}{|l|c|c|c|}
\hline \multicolumn{1}{|c|}{ Modelo } & $\begin{array}{c}\text { MAPE ou } \\
\text { EPAM }\end{array}$ & $\begin{array}{c}\text { MAD ou } \\
\text { EAM }\end{array}$ & $\begin{array}{c}\text { MSD ou } \\
\text { EQM }\end{array}$ \\
\hline Média Móvel & 71,0 & 114,7 & 18535,0 \\
\hline Suavização Exponencial Simples & 42,2 & 95,8 & 14761,0 \\
\hline Suavização Exponencial Dupla & 41,4 & 101,2 & 16448,5 \\
\hline Suavização Winters Multiplicativa & 39,7 & 102,7 & 16939,1 \\
\hline Suavização Winters Aditiva & 39,1 & 101,9 & 16910,3 \\
\hline ARIMA(1,1,1) & 32,8 & 103,7 & 17541,6 \\
\hline ARIMA(2,1,1) & 31,1 & 89,1 & 14806,6 \\
\hline ARIMA(3,2,2) & 49,0 & 142,2 & 30801,9 \\
\hline ARIMA(3,1,3) & 29,7 & 87,7 & 13448,0 \\
\hline
\end{tabular}

Com relação ao modelo ARIMA, foram aplicados com outros parâmetros p,d e q, porém estes apresentam erro semelhante aos 4 modelos da tabela 12. O modelo ARIMA (3,2,2), mesmo apresentando MAPE consideravelmente maior que os outros modelos ARIMA, foi mantido na tabela para deixar claro que uma combinação entre os parâmetros destes modelos é o ponto fundamental para a escolha do melhor modelo. As técnicas de obtenção dos parâmetros não são citadas neste trabalho, já que o foco é a aplicação da ferramenta matemática, com auxílio da ferramenta computacional, com a análise e interpretação dos dados.

\section{Conclusão}

As previsões geradas pelo modelo ARIMA $(3,1,3)$ apresentam erro percentual absoluto médio e erro absoluto médio menor que os mesmos tipos de erros gerados pelos outros modelos. Isto se deve ao fato de que os modelos ARIMA são mais apropriados quando a série histórica apresenta componentes de tendência e sazonalidade, características que os modelos de médiamóvel, por exemplo, não dão tratamento adequado. Os modelos de ajuste de tendência linear e quadrático, também apresentaram bons resultados, e levam uma vantagem com relação aos modelos ARIMA, por serem mais fáceis de aplicar e interpretar os resultados.

No caso do modelo de médias móveis, utilizado pela empresa ate o momento, além 
de não ser adequado para gerar previsões com a curva de demanda, que possui componentes de tendência e sazonalidade, apresenta MAPE muito elevado, em torno de $70 \%$.

Os modelos de suavização, por conterem constantes que ajustam as componentes de sazonalidade e/ou tendência apresentaram resultados razoáveis, e também são de fácil aplicação e interpretação. No momento atual da empresa, seria mais interessante a adoção destes modelos (ajuste de tendências ou suavização) em substituição aos modelos de médias-móveis, pois apresentam muita semelhança na aplicação, e seus resultados estão próximos aos dos demais modelos. Num outro momento a implantação dos modelos ARIMA seria a decisão ideal, pois apresentam menor erro, e ainda é possível obter maiores níveis de melhoria na previsão ao encontrar os parâmetros (p,d,q) ideais. Possivelmente obtendo erros menores do que o apresentado na tabela 17. Para a obtenção dos parâmetros ideais será necessário um estudo teórico mais aprofundado na modelagem com o ARIMA, pois o presente trabalho focou na busca de menores erros de previsão com a utilização do software Minitab ${ }^{\circledR}$.

Os dados apresentados neste trabalho mostram que é perfeitamente possível a melhoria da previsão de demanda com o fornecimento de informações mais precisas ao setor de planejamento e controle da produção, o PCP, gerando resultados positivos não somente neste setor, mas como em toda a organização. Diversas tomadas de decisão serão possíveis com a previsão de demanda realizadas com taxas de erros menores.

$$
\text { Recomenda-se que a empresa }
$$
introduza métodos científicos de previsão no auxílio à tomada de decisão, tanto no processo de avaliação da demanda, quanto em outros processos. É importante que a empresa tenha a compreensão de que o método científico não irá fornecer as respostas para os problemas encontrados, mas mostrarão aspectos importantes na hora de decidir. Além disso, permitirá traçar um caminho fácil de ser seguido em busca dos objetivos da empresa, como aumento do lucro, alcançar as metas de vendas, diminuição de não conformidades dos produtos, diminuição de custos de produção e etc.

A utilização de ferramentas estatísticas, se difundida na empresa, criará um diferencial fundamental na tomada de decisão, pois esta não ficará dependente somente do julgo do administrador, sua experiência e conhecimento. Haverá informações importantes para a melhor tomada de decisão. É interessante também que nas reuniões de previsão de demanda estejam presentes os demais setores da empresa, já que uma previsão de demanda feita com qualidade necessita de informações de todos os setores. Isso irá refletir também de forma positiva no desempenho de todos os setores. 
É importante também que ocorra o rompimento de algumas barreiras culturais com relação à utilização de métodos científicos nas empresas. Ainda perdura a visão do gestor conhecedor do mercado ou processo, com larga experiência e conhecimento, que toma as decisões operacionais ou estratégicas com base apenas no feeling. Hoje com a velocidade das mudanças nas empresas, economia e mercados, nenhum grau de conhecimento ou experiência será mais rápido e eficiente do que a análise de informações em softwares estatísticos e matemáticos.

Além da agilidade e acurácia que os métodos estatísticos podem fornecer ao tomador de decisão, este poderá perceber mudanças na demanda dos produtos da empresa antes que isto seja alarmado por crises financeiras e de mercado. Ao analisarmos a curva de demanda do produto A, no período de janeiro de 2007 a dezembro de 2011 vemos uma tendência positiva, ou seja, crescimento nas vendas.

\section{REFERÊNCIAS}

BERTOLO, L.A.Técnicas de previsão de Box- Jenkins no Excel, IMES - Catanduva, 2010.

CHASE, R.B.; AQUILANO, N.J.; JACOBS, F.R. Administração da produção para vantagem competitiva, 11 ed, São Paulo, McGrawHill, 2010.

CORREA, H.; GIANESI, I.; CAON, M.; Planejamento, programação e controle da Produção, 5 ed. São Paulo, Atlas, 2009.
COSTA, M; JACOBS, WILLIAM; Previsão da demanda de um produto industrial utilizando a metodologia de Box-Jenkin.

Revista Destaques acadêmicos, CETEC/UNIVATES, v.3, n.4, 2011.

FAVA, V. L. Manual de econometria. In: VASCONCELOS, M. A. S.; ALVES, D. São Paulo: Editora Atlas, 2000.

FELIN NUNES, C.; WEISE, A.D.; SOUTO BOLZAN MEDEIROS, F.. Uma proposta de alinhamento das áreas de pesquisa em um programa de Pós-Graduação em Engenharia de Produção. Revista Produção e

Desenvolvimento, v. 1, n. 2, p. 44-55, 2015.

GALVÍNCIO, J.D; SALVIANO de Sousa, F.D; SOUZA, I.F; Uso de modelos autoregressivos e médias-móveis para geração de vazões médias mensais na bacia hidrográfica do alto São Francisco, XII Congresso Brasileiro de Meteorologia, Foz de Iguaçu-PR, 2002.

GIL, Antonio Carlos. Métodos e técnicas de pesquisa social. São Paulo: Atlas, 1999

HASTENTEUFEL, C., LARENTIS, F. Análise da rentabilidade de clientes através da margem de contribuição: um estudo em uma empresa de médio porte do setor moveleiro localizada na Serra Gaúcha. Revista Produção e Desenvolvimento, v.1, n.1, p.60-76, 2015.

LEMOS, F.O. Metodologia para seleção de métodos de previsão de demanda, Dissertação para obtenção de título de mestre, UFRGS, Porto Alegre, 2006.

LUSTOSA, L.; MESQUITA, M.; QUELHAS, R.; Planejamento e controle da Produção, 1 ed. Rio de Janeiro, Elsevier, 2008.

MARTINS, P.G.; LAUGENI, F.P.; Administração da produção,2ed, São Paulo, Saraiva, 2010.

MOREIRA,E.;PACAGNELLA JÚNIOR, A.C.; PACİFICO, O.; SALGADO JÚNIOR, 
A. P. Contribuições do planejamento e controle da produção para a competitividade empresarial: um estudo em uma empresa do setor moveleiro. Espacios, v.35, n.9, 2014.

MORETTIN, P.A; TOLOI, C.M.Análise de séries temporais, 2ed ver ampl, São Paulo, Blucher, 2006.

PETRÔNIO, G. M; LAUGENI, P. F.

Administração da Produção. $2^{a}$ ed. São Paulo. Ed.Saraiva. 2005

SENNA, P., TANSCHEIT, R., GOMES, A.M. Planejando o processo de previsão de demanda com auxílio da lógica fuzzy. Revista Produção e Desenvolvimento, v.1, n.2, p.90103, mai/ago, 2015.

SILVA, E.L; MENEZES, E.M; Metodologia de pesquisa e elaboração de dissertação, 4ed, Florianópolis, UFSC, 2005.

SOUZA, G.P.; SAMOHYL, R.W.; DE MIRANDA, R.G.; Métodos simplificados de previsão empresarial, 1 ed. Rio de Janeiro, Ciencia Moderna, 2008.

SOUZA, R.C; CAMARGO, M.E.; Análise e previsão de séries temporais; Os modelos ARIMA, 2ed, Rio de Janeiro, 2004.

TUBINO, D.F.; Planejamento e controle da produção - Teoria e Prática, 1ed, 2 reimpr., São Paulo, Atlas, 2008.

VOLLMANN, T.E.; BERRY, W.L.;

WHYBARK, D.C.; JACOBS, F.R.; Sistemas de planejamento e controle da produção para o gerenciamento da cadeia de suprimentos, 5ed., São Paulo, Bookman, 2005. 